\title{
Rapid Preparation of Subfractions of Low Density Lipoprotein from Hypercholesterolemic Rabbit Serum
}

\author{
Tomohiro YamauchI, Taisuke InAGaki, Nobuko OHISHI, \\ and Kunio YAGI* \\ Institute of Applied Biochemistry, Yagi Memorial Park, \\ Mitake, Gifu 505-01, Japan
}

(Received August 24, 1989)

\begin{abstract}
Summary A method for rapid preparation of subfractions of low density lipoprotein (LDL) from the serum of cholesterol-fed rabbits was devised. The method includes three steps: (1) removal of chylomicrons and very low density lipoprotein by ultracentrifugation with a vertical rotor for $1 \mathrm{~h}$; (2) separation of LDL subfractions, LDL-1, LDL-2, and LDL-3, by ultracentrifugation with a vertical rotor for $2 \mathrm{~h}$; and (3) Fast Protein, Polypeptide, Polynucleotide Liquid Chromatography (FPLC) for purification of these LDL subfractions. The whole procedure of the present method could be completed within $13 \mathrm{~h}$. On the basis of size and density distribution, LDL-1 corresponded to intermediate density lipoprotein obtained by the standard sequential floatation method ( $d=$ $1.006-1.019$ ) from the same serum. Gel electrophoresis, density distribution, and FPLC analysis showed that LDL-1 was contaminated with neither other lipoproteins nor serum proteins. LDL-3 was found to contain LDL obtained by the previously reported method from the serum of a rabbit fed a normal diet. LDL-2 had properties intermediate between those of LDL-1 and LDL-3.
\end{abstract}

Key Words: low density lipoprotein subfraction, hypercholesterolemic serum, discontinuous centrifugation, FPLC, rabbit

Serum from cholesterol-fed rabbits is characterized by a marked increase in the contents of very low density lipoprotein (VLDL) and chylomicrons as well as low density lipoprotein (LDL) and has been used for studies on the relationship between hyperlipidemia and the development of atherosclerosis. In such studies,

*To whom correspondence should be addressed. 
the preparation of intermediate density lipoprotein (IDL) and LDL has been made by the standard sequential floatation method [1]. However, this method is a time-consuming one and the preparations are contaminated with VLDL and chylomicrons. In addition, a lengthy ultracentrifugation results in the release of apoprotein $\mathbb{E}$ (Apo $E$ ) from the surface of lipoprotein particles $[2,3]$. Other preparative methods with a swinging bucket rotor $[4,5]$ also possess the same shortages as described above.

In order to overcome these disadvantages of the standard method, a rapid method for the preparation of less denatured LDL species from hyperlipidemic serum is required. Previously, we devised a rapid method for the isolation of purer and less denatured LDL from the serum of a rabbit fed a normal diet by a combination of discontinuous density gradient ultracentrifugation in a vertical rotor and Fast Protein, Polypeptide, Polynucleotide Liquid Chromatography (FPLC) [6]. In the present study, we applied this method to the serum of the hypercholesterolemic rabbit, and describe the rapid preparation of subfractions of purer and less denatured LDL from such serum.

\section{MATERIALS AND METHODS}

Serum. Blood was collected from Japanese white rabbits that had been fed a commercial diet (CE-3, Clea Japan, Inc., Tokyo) supplemented with $2 \%$ cholesterol for 2 weeks. For coagulation, blood was stood overnight at $4^{\circ} \mathrm{C}$, and the serum was then collected.

Ultracentrifugation in a vertical rotor and FPLC. Ultracentrifugation in a vertical rotor (Hitachi RPV 50T) was carried out according to the method of Chung et al. [7] in a Beckman L8-55 ultracentrifuge at 50,000 rpm for $2 \mathrm{~h}$ at $10^{\circ} \mathrm{C}$ with the slow start setting and without braking. Afterwards, $0.53-\mathrm{ml}$ fractions were collected from the top by use of a Density Gradient Fractionator DGF-U (Hitachi Koki Co., Ltd., Tokyo), and an aliquot of each fraction was used for determination of cholesterol. FPLC was performed on a Superose 6 column $(10 \times 300 \mathrm{~mm})$ (Pharmacia Fine Chemicals, Uppsala), as described $[6,8]$. Sample was applied onto a column of the FPLC system previously equilibrated with $10 \mathrm{~mm}$ Tris- $\mathrm{HCl}$ buffer ( $\mathrm{pH}$ 7.4) containing $0.15 \mathrm{M} \mathrm{KCl}$ and $1 \mathrm{~mm}$ EDTA; and the sample was eluted with the same buffer at a flow rate of $0.5 \mathrm{ml} / \mathrm{min}$. Fractions of $0.25 \mathrm{ml}$ were collected, and the cholesterol content in each fraction was determined.

Preparation of chylomicron- and VLDL-deficient serum. Eighteen milliliters of saline containing $0.01 \%$ EDTA was layered over $15 \mathrm{ml}$ of serum. After ultracentrifugation in a vertical rotor at $50,000 \mathrm{rpm}$ for $1 \mathrm{~h}$ at $10^{\circ} \mathrm{C}$ with the slow start setting and without braking, the top $18 \mathrm{ml}$, which was checked by FPLC to contain no lipoproteins and serum proteins other than chylomicrons and VLDL, was removed; and the bottom fraction $(15 \mathrm{ml})$ was used as chylomicron- and VLDL-deficient serum. 
Preparation of IDL and LDL by the standard method. IDL and LDL were isolated from the serum of a cholesterol-fed rabbit at density intervals of $1.006-1.019$ and $1.019-1.063$, respectively, according to the standard sequential floatation method [1]. Each lipoprotein preparation obtained was then dialyzed against $0.15 \mathrm{M} \mathrm{NaCl}$ containing $1 \mathrm{~mm}$ EDTA for $24 \mathrm{~h}$ at $4^{\circ} \mathrm{C}$. For the comparison of particle size and density distribution, we also prepared LDL from the serum of a rabbit fed a normal diet by our method reported previously [6] and used it as normal LDL.

Concentration of sample. Solutions containing lipoprotein were concentrated with Centriflow CF50A filter cones (Amicon Corp., Danvers, MA).

Analytical procedures. For determination of the density of final preparations, samples were dialyzed for $24 \mathrm{~h}$ at $4^{\circ} \mathrm{C}$ against $\mathrm{KBr} / \mathrm{NaCl}$ solution $(d=1.040)$ containing $0.01 \%$ EDTA to adjust their density to $1.040 \mathrm{~g} / \mathrm{ml}$. Five milliliters of $\mathrm{KBr} / \mathrm{NaCl}$ solution $(d=1.080)$ containing $0.01 \%$ EDTA was overlaid consecutively with $4 \mathrm{ml}$ of sample $(d=1.040), 15 \mathrm{ml}$ of $\mathrm{KBr} / \mathrm{NaCl}$ solution $(d=1.020)$ containing $0.01 \%$ EDTA, and then $10 \mathrm{ml}$ of saline containing $0.01 \%$ EDTA. Ultracentrifugation was performed in a vertical rotor at $50,000 \mathrm{rpm}$ for $3 \mathrm{~h}$ at $10^{\circ} \mathrm{C}$ with the slow start setting and without braking. After ultracentrifugation, $0.53-\mathrm{ml}$ fractions were collected as described above, and the cholesterol content in each fraction was determined. The density of each fraction was measured with a Density/Specific Meter DA-210 (Kyoto Electronics Manufacturing Co. Ltd., Kyoto).

For determination of size distribution of the final preparations, samples were applied onto a Superose 6 column of the FPLC system under the same conditions as described above. Elution pattern was determined by measurement of the content of cholesterol in each fraction.

Agarose gel electrophoresis was performed with 1\% agarose gels (Corning Medical, Palo Alto, CA), and sodium dodecyl sulfate-polyacrylamide gel electrophoresis (SDS-PAGE) was carried out according to the method of Laemmli [9]. SDS-PAGE standards of low and high molecular weight (Bio-Rad Laboratories, Richmond, CA) were used as marker proteins.

Contents of cholesterol, triglyceride, and phospholipids were determined enzymatically with the use of Determiner TC 555, Determiner TG-S 555, and Determiner PL (Kyowa Medex Co. Ltd., Tokyo), respectively. Protein content was determined by the method of Markwell et al. [10]. Apoprotein B (Apo B) content was determined as the difference between the soluble protein content of a sample treated with $4.2 \mathrm{M} \mathrm{1,1,3,3-tetramethylurea} \mathrm{and} \mathrm{that} \mathrm{of} \mathrm{an} \mathrm{untreated} \mathrm{one} \mathrm{[11].} \mathrm{Apo}$ E content was assigned to 1,1,3,3,-tetramethylurea-soluble protein, because LDL samples were found to contain only apo B and apo E, but not other apoproteins or serum proteins. Lipid peroxide level was determined by the hemoglobinmethylene blue method $[12,13]$. 


\section{RESULTS AND DISCUSSION}

\section{Separation of LDL subfractions}

The density of chylomicron- and VLDL-deficient serum was adjusted to 1.25 $\mathrm{g} / \mathrm{ml}$ with solid $\mathrm{KBr}$. After $30 \mathrm{ml}$ of saline containing $0.01 \%$ EDTA had been layered over $5 \mathrm{ml}$ of the above serum, ultracentrifugation followed by fractionation was performed as described in Materials and Methods. Cholesterol, triglyceride, and protein contents in each fraction were determined. As shown in Fig. 1, the fractions from numbers 15 to 50 were found to contain LDL, which was clearly separated from small peaks of VLDL (tube numbers 1-5), high density lipoprotein (HDL) and serum proteins (following tube number 58). The LDL fraction appeared to be separated into at least three peaks. Accordingly, fractions in tube numbers 18-30, 31-37, and 38-47 were separately pooled and designated as crude LDL-1, LDL-2, and LDL-3, respectively.

Each crude LDL subfraction was applied onto a Superose 6 column of the FPLC system in order to remove contaminants and $\mathrm{KBr}$, as described in Materials and Methods. As shown in Fig. 2, crude LDL-1 as well as LDL-2 contained a small amount of VLDL, and crude LDL-3, a large amount of serum proteins. The peak fractions of each sample shown by bar in Fig. 2 were pooled, concentrated with Centriflow CF50A filter cones, and designated purified LDL-1, LDL-2, and LDL-3. The purified LDL subfractions were kept in an ice until used.

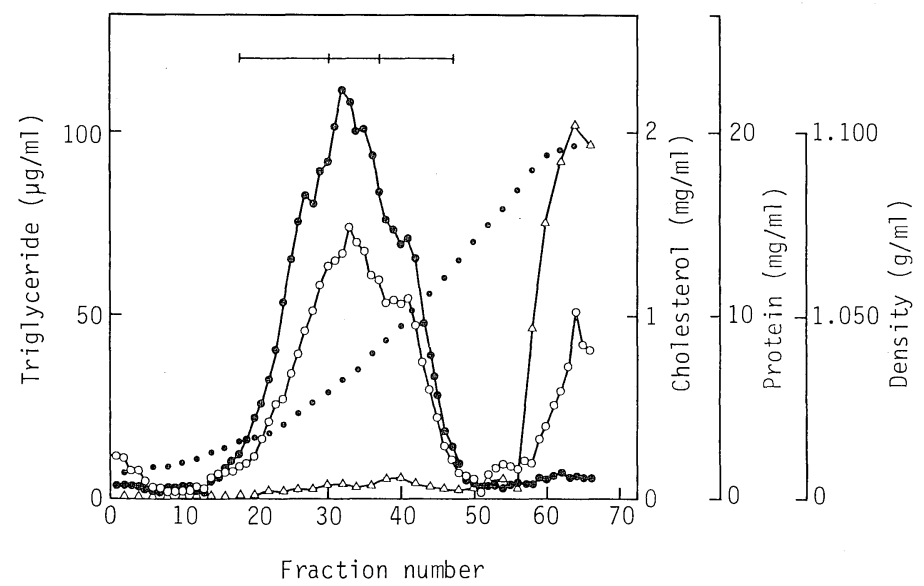

Fig. 1. Density-dependent distribution of lipoproteins of chylomicron- and VLDLdeficient serum from a cholesterol-fed rabbit after ultracentrifugation with a vertical rotor. Ultracentrifugation and detection of lipoprotein were carried out as described in the text. LDL-1, LDL-2, and LDL-3 were collected separately as indicated by the bar. Open circles, triglyceride; closed circles, cholesterol; open triangles, protein. Dots represent density of fractions. 


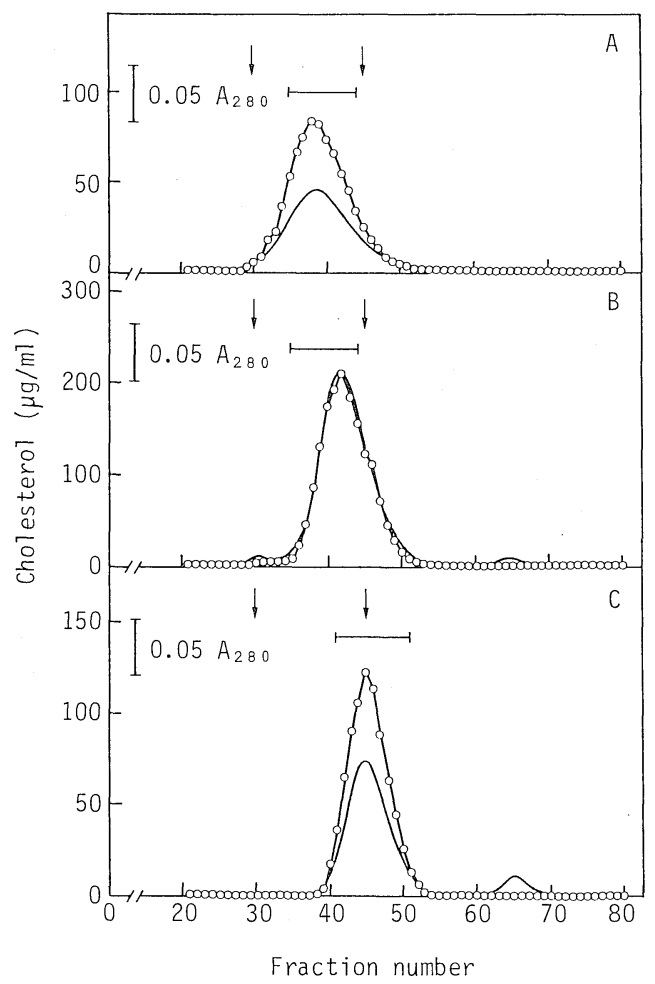

Fig. 2. Elution profiles of crude LDL subfractions from the Superose 6 column of the FPLC system. A, Crude LDL-1; B, crude LDL-2; C, crude LDL-3. Solid line, absorbance at $280 \mathrm{~nm}$. Left arrow indicates the elution peak of VLDL; and the right arrow, that of LDL.

\section{Purity and properties of LDL subfractions}

Each LDL subfraction obtained by the present method was compared with IDL and LDL obtained by the standard method in terms of the density distribution, distribution of particle size determined by the FPLC system, agarose gel electrophoresis, SDS-PAGE, and chemical composition.

Figure 3 shows the density distribution of the purified LDL subfractions (A), IDL and LDL obtained by the standard method (B), and normal LDL isolated by our previously reported method (C). LDL-1 isolated by the present method appears to correspond to IDL. Purified LDL-3 was found to be involved in LDL obtained by the standard method, which contains a large amount of IDL. This may be attributable to convective disturbance and adherence of lipoprotein to the wall of the centrifuge tube. It should be noted that both peaks of LDL-3 and LDL obtained by the standard method have a shoulder at density 1.035, which might be ascribed to the peak of LDL prepared by our previous method [6] from a rabbit fed a normal diet. LDL-2 showed a density distribution between those of LDL-1 and LDL-3. 


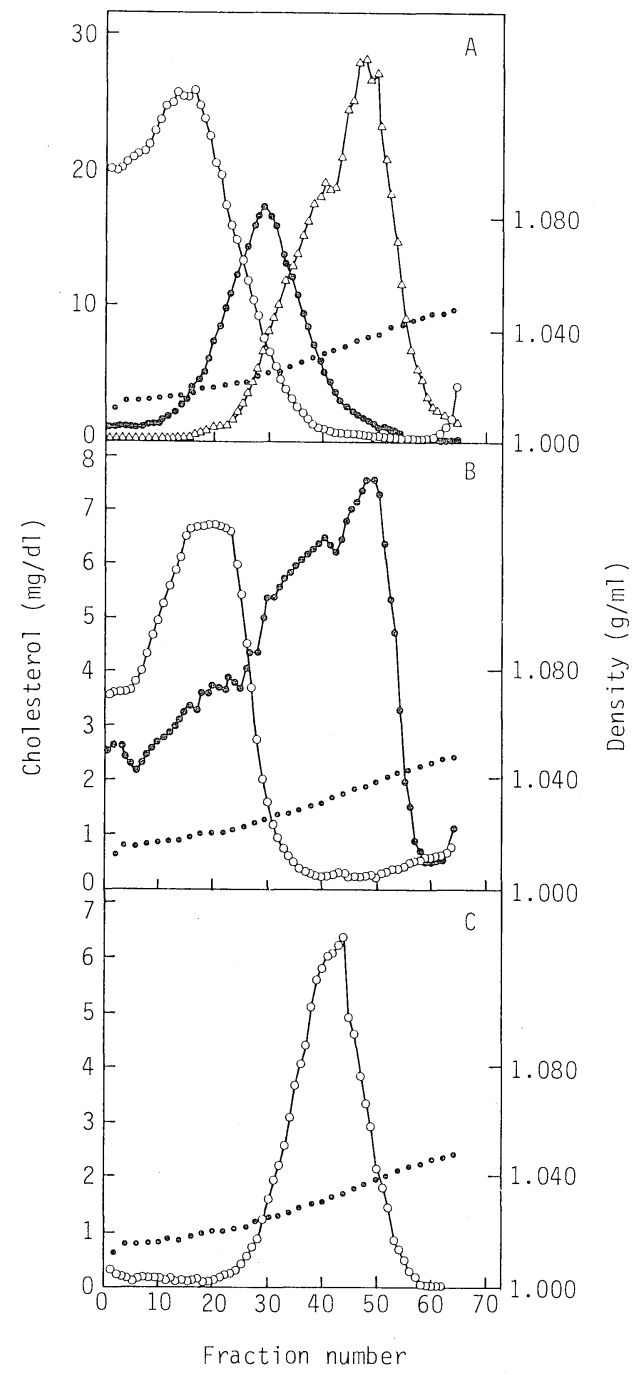

Fig. 3. Density distribution of the purified LDL subfractions, of IDL and LDL obtained by the standard method, and of normal LDL. The density gradient ultracentrifugation was performed as described in the text. Patterns are shown in terms of cholesterol concentration. A, LDL subfractions isolated by the present method: open circles, LDL-1; closed circles, LDL-2; open triangles, LDL-3. B, IDL and LDL isolated from serum of a cholesterol-fed rabbit by the standard method: open circles, IDL; closed circles, LDL. C, LDL isolated from normal rabbit serum by our method reported previously [6]. Dots represent density of fractions.

The FPLC elution profiles of the purified LDL-1, LDL-2, and LDL-3 were compared with those of IDL and LDL obtained by the standard method. As shown in Fig. 4, each subfraction was eluted as a single and symmetrical peak. So 


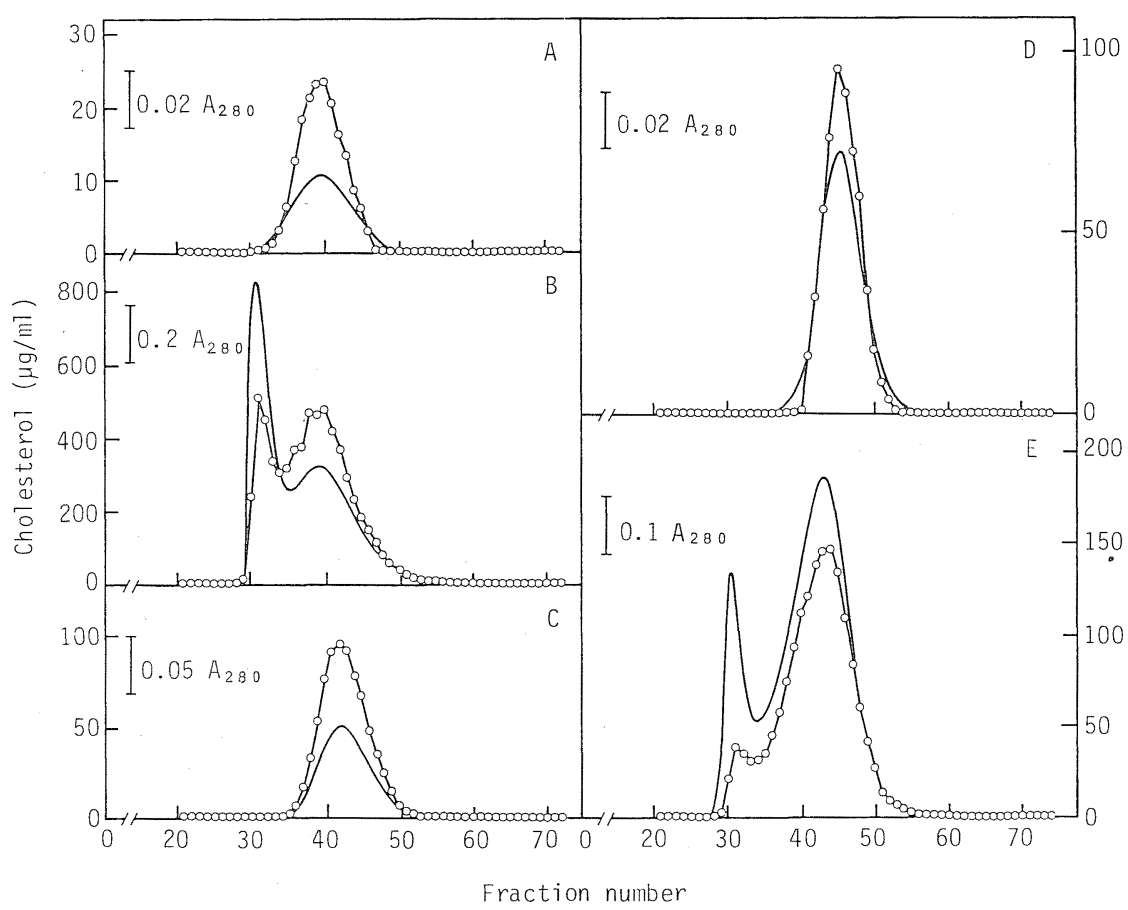

Fig. 4. Elution profiles of the purified LDL subfractions and of IDL and LDL obtained by the standard method on the Superose 6 column of the FPLC system. A, LDL-1; B, IDL isolated by the standard method; C, LDL-2; D, LDL-3; E, LDL isolated by the standard method. Open circles, cholesterol. Solid line, absorbance at $280 \mathrm{~nm}$.

far as judged from their elution patterns, they seem to be contaminated with neither other lipoproteins such as VLDL and HDL nor serum proteins (see Fig. $4 \mathrm{~A}, \mathrm{C}$, and D). However, IDL isolated by the standard method was contaminated with large amounts of chylomicrons and VLDL as shown in Fig. 4 B, and the sample of LDL obtained by the standard method contained large amounts of VLDL and IDL, causing the shift of its peak toward a larger particle size area (Fig. $4 \mathrm{E})$.

As shown in Fig. 5, a single band migrating to the $\beta$-position was observed on an agarose gel for each of purified LDL-1, LDL-2, and LDL-3, as well as for IDL and LDL obtained by the standard method. For examination of the purity of IDL and LDL isolated from hypercholesterolemic rabbit serum, agarose gel electrophoresis is not appropriate, since the electrophoretic mobility of the VLDL in such serum changes from a pre $\beta$-to $\beta$-position on the gel, as described by Shore et al. [14] and Stange et al. [15]. In fact, both IDL and LDL obtained by the standard method gave a single band on the gel, though density distribution and FPLC analysis showed that they were contaminated with other lipoproteins.

SDS-PAGE analysis showed that purified LDL-1, LDL-2, and LDL-3 Vol. 8, No. 1, 1990 


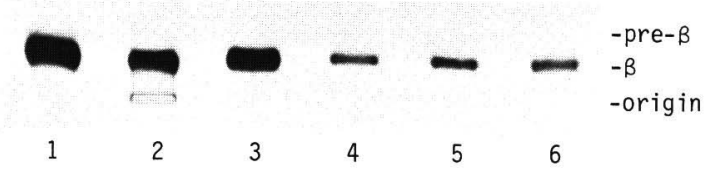

Fig. 5. Agarose gel electrophoresis of the purified LDL-1, LDL-2, and LDL-3, and of IDL and LDL obtained by the standard method. Lane 1, whole serum obtained from a cholesterol-fed rabbit; lane 2, IDL obtained by the standard method; lane 3, LDL obtained by the standard method; lane 4, LDL-1; lane 5, LDL-2; lane 6, LDL-3. The positions of pre- $\beta$ and $\beta$ were determined with normal rabbit serum. The gel was stained with Sudan Black B.

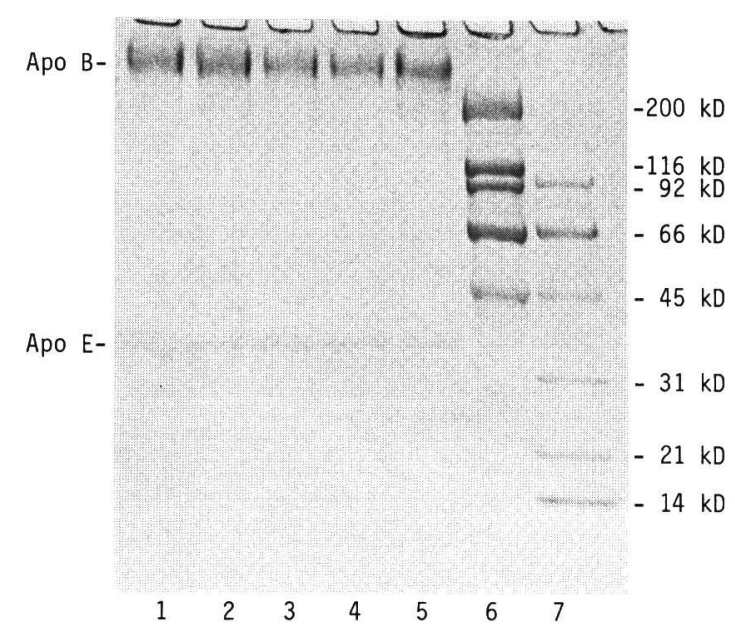

Fig. 6. SDS polyacrylamide gradient gel electrophoresis of apolipoproteins of the purified LDL-1, LDL-2, and LDL-3, and of IDL and LDL obtained by the standard method. Lane 1. IDL; lane 2, LDL; lane 3, LDL-1; lane 4, LDL-2; lane 5, LDL-3; lane 6, marker proteins (high molecular weight); lane 7 , marker proteins (low molecular weight).

contained both apo B and apo $\mathrm{E}$ but not other apoproteins (Fig. 6), indicating no contamination with HDL. Then, we determined apo $\mathrm{B}$ and apo E contents in the purified LDL subfractions by the procedure stated in Materials and Methods. The ratio of apo $\mathrm{E} /$ apo $\mathrm{B}$ was $2.33,1.53$, and 0.18 for the LDL-1, LDL-2, and LDL-3 fraction, respectively.

Table 1 shows the chemical composition of the purified LDL-1, LDL-2, and LDL-3, and of IDL and LDL obtained by the standard method. The sum of the contents of cholesterol, triglyceride, phospholipids, and protein of IDL plus LDL prepared by the standard method was higher than that of the combined LDL subfractions (LDL-1, LDL-2, and LDL-3) isolated by the present method. This can be ascribed to the contamination with VLDL when the standard method is employed. The content of the total protein in the former was lower than that of the latter. The apo B content of the former $(71.0 \mathrm{mg} / \mathrm{dl})$ did not differ from that of the 
Table 1. Chemical composition of LDL subfractions obtained by the present method and of IDL and LDL separated by the standard method.

\begin{tabular}{|c|c|c|c|c|c|}
\hline \multirow{2}{*}{ Component } & \multicolumn{3}{|c|}{ Present method } & \multicolumn{2}{|c|}{ Standard method } \\
\hline & LDL-1 & LDL-2 & LDL-3 & IDL & LDL \\
\hline Cholesterol & $53.6 \pm 4.2$ & $49.8 \pm 4.4$ & $37.5 \pm 3.1$ & $64.9 \pm 8.2$ & $129.5 \pm 10.6$ \\
\hline Triglyceride & $4.3 \pm 1.5$ & $5.1 \pm 1.7$ & $4.1 \pm 1.3$ & $2.0 \pm 0.4$ & $5.4 \pm 1.5$ \\
\hline Phospholipids & $23.7 \pm 1.9$ & $23.2 \pm 1.1$ & $20.0 \pm 0.9$ & $27.2 \pm 2.9$ & $60.1 \pm 7.3$ \\
\hline Protein & $26.5 \pm 3.2$ & $33.6 \pm 4.0$ & $34.8 \pm 1.4$ & $22.5 \pm 4.2$ & $57.7 \pm 7.4$ \\
\hline Total & $103.1 \pm 3.7$ & $111.7 \pm 5.5$ & $96.4 \pm 3.0$ & $116.5 \pm 13.0$ & $252.6 \pm 25.1$ \\
\hline
\end{tabular}

All values represent $\mathrm{mg} / \mathrm{dl}$ serum. Means $\pm \mathrm{SE}$ of 4 experiments are given.

Table 2. Ratio of lipid peroxide level to total lipids in LDL subfractions.

$\begin{array}{ccc}\text { Standard method } & \text { IDL } & 0.77 \pm 0.10 \\ & \text { LDL } & 0.40 \pm 0.06 \\ \text { Present method } & \text { LDL-1 } & 0.38 \pm 0.03^{*} \\ & \text { LDL-2 } & 0.31 \pm 0.06 \\ & \text { LDL-3 } & 0.26 \pm 0.07\end{array}$

Means $\pm \mathrm{SE}$ of the ratio of lipid peroxides $(\mathrm{nmol} / \mathrm{dl})$ to total lipids $(\mathrm{mg} / \mathrm{dl})$ of $\mathrm{LDL}$ fraction of 3 experiments are given. ${ }^{*}$ Statistically different from IDL $(p<0.05)$.

latter $(68.7 \mathrm{mg} / \mathrm{dl})$, indicating that the apo E content in the total IDL and LDL obtained by the standard method was smaller than that in the total LDL-1, LDL-2, and LDL-3. As pointed out by Blum et al. [2] and Mackie [3], apo E is susceptible to release from the surface of the lipoprotein particle during extended ultracentrifugation. Thus, the lower content of apo E in IDL and LDL prepared by the standard method can be ascribed to the lengthy ultracentrifugation used in the method. Further, it can be expected that the denaturation of the preparation due to long exposure to oxygen can be reduced in the present method. Table 2 summarizes the data of lipid peroxide levels of the present samples and those of IDL and LDL obtained by the standard method. It is especially notable that the level of LDL-1 is obviously lower than that of IDL obtained by the standard method. Judging from the data in Table 2, we can conclude that the present preparations are less denatured compared with those prepared by the standard method. This conclusion is also supported by the data of lipoprotein analysis mentioned above.

All the results described in the present paper indicate that the present method is time saving; i.e., the whole procedure can be completed within $13 \mathrm{~h}$, as summarized in Scheme 1, whereas it takes 4 days in the case of the standard method. Additionally, the final products isolated by the present method are less denatured and much purer; LDL-1, LDL-2, and LDL-3 were contaminated with neither other lipoproteins nor serum proteins. Accordingly, LDL species obtained by the present method from hypercholesterolemic serum should be useful for studies on atherogenesis.

Vol. 8, No. 1, 1990 
Procedure

Hypercholesterolemic serum, $15 \mathrm{ml}$

$\longleftarrow$ layer saline containing $0.01 \%$ EDTA, $18 \mathrm{ml}$

$\longleftarrow$ centrifuge at $50,000 \mathrm{rpm}$ for $1 \mathrm{~h}$ (slow accelerating and without braking)

$\longleftarrow$ remove top $18 \mathrm{ml}$

$\longleftarrow$ collect bottom fraction $(15 \mathrm{ml})$

Chylomicron- and VLDL-deficient serum

$\longleftarrow$ adjust to $d=1.25$ with solid $\mathrm{KBr}, 5 \mathrm{ml}$

$\longleftarrow$ layer saline containing 0.01\% EDT A, $30 \mathrm{ml}$

$\longleftarrow$ centrifuge at $50,000 \mathrm{rpm}$ for $2 \mathrm{~h}$

(slow accelerating and without braking)

$\longleftarrow$ collect fractions with a gradient fractionator (each fraction, $0.53 \mathrm{ml}$ )

fraction numbers: crude LDL-1, 18-30; crude LDL-2,

31-37; crude LDL-3, 38-47

Crude LDL subfractions

$\longleftarrow$ concentrate with a Centriflow CF50A

« apply onto a Superose 6 column of FPLC system

$\longleftarrow$ elute with $10 \mathrm{~mm}$ Tris- $\mathrm{HCl}$ buffer ( $\mathrm{pH}$ 7.4) containing

$0.15 \mathrm{~mm} \mathrm{KCl}$ and $1 \mathrm{~mm}$ EDTA at a flow rate of $0.5 \mathrm{ml} / \mathrm{min}$

(each fraction, $0.25 \mathrm{ml}$ )

Fractions containing each LDL subfraction

$\longleftarrow$ concentrate with a Centriflow CF50A

LDL subfractions

Total 13.0

Scheme 1. Isolation of LDL subfractions from hypercholesterolemic rabbit serum.

This work was supported in part by a Grant-in-Aid (63113003) from the Ministry of Education, Science and Culture of Japan.

\section{REFERENCES}

1. Hatch, F.T., and Lees, R.S. (1968): Practical methods for plasma lipoprotein analysis. $A d v$. Lipid Res., 6, 1 -68.

2. Blum, C.B., Aron, L., and Sciacca, R. (1980): Radioimmunoassay studies of human apolipoprotein E. J. Clin. Invest., 66, 1240-1250.

3. Mackie. A. (1981): Concentration and distribution of human plasma apo E. Clin. Chim. Acta, 116, 35-41

4. Shen, M.M.S., Krauss, R.M., Lindgen, F.T., and Forte, T.M. (1981): Heterogeneity of serum low density lipoproteins in normal human subjects. J. Lipid Res., 22, 236-244.

5. Swinkel, D.W., Hak-Lemmers, H.L.M., and Demacker, P.N.M. (1987): Single spin density gradient ultracentrifugation method for the detection and isolation of light and heavy low density lipoprotein subfractions. J. Lipid Res., 28, 1223-1239.

6. Yamauchi, T., Inagaki, T., Ohishi, N., and Yagi, K. (1989): Rapid preparation of low 
density lipoprotein from normal rabbit serum. J. Clin. Biochem. Nutr., 7, 133-141.

7. Chung, B.H., Wilkinson, T., Geer, J.C., and Segrest, J.P. (1980): Preparative and quantitative isolation of plasma lipoproteins; rapid, single discontinuous density gradient ultracentrifugation in a vertical rotor. J. Lipid Res., 21, 284-291.

8. Ha, Y.C., and Barter, P.J. (1985): Rapid separation of plasma lipoproteins by gel permeation chromatography on agarose gel Superose 6B. J. Chromatogr., 341, 154-159.

9. Laemmli, U.K. (1970): Cleavage of structural proteins during the assembly of the head of bacteriophage-T4. Nature, 227, 680-685.

10. Markwell, M.A.K., Haas, S.M., Tolbert, N.E., and Bieber, L.L. (1981): Protein determination in membrane and lipoprotein samples: Manual and automated procedures. Methods Enzymol., 72, 296-303.

11. Kane, J.P., Sata, T., Hamillon, R.L., and Havel, R.J. (1975): Apoprotein composition of very low density lipoproteins in human serum. J. Clin. Invest., 56, 1622-1634.

12. Ohishi, N., Ohkawa, H., Miike, A., Tatano, T., and Yagi, K. (1985): A new assay method for lipid peroxides using a methylene blue derivative. Biochem. Int., 10, 205-211.

13. Yagi, K., Kiuchi, K., Saito, Y., Miike, A., Kayahara, N., Tatano, T., and Ohishi, N. (1986): Use of a new methylene blue derivative for determination of lipid peroxides in food. Biochem. Int., 12, 367-371.

14. Shore, V.G., Shore, B., and Hart, R.G. (1974): Changes in apolipoproteins and properties of rabbit very low density lipoproteins on induction of cholesterolemia. Biochemistry, 13 , 1579-1584.

15. Stange, E., Agostini, B., and Papaerberg, J. (1975): Changes in rabbit lipoprotein properties by dietary cholesterol and saturated and polyunsaturated fats. Atherosclerosis, 22, 125-148. 